\title{
Influence du complément alimentaire d'asticots frais sur la productivité des poules locales et le revenu des aviculteurs au Sud-Ouest du Bénin
}

\author{
Edenakpo K. Aimé $^{* 2}$, Houndonougbo P. Venant ${ }^{1}$, Ahoyo Adjovi N. René ${ }^{3}$ Behingan M. \\ Boris $^{1}$, Houndonougbo M. Frédéric ${ }^{1}$, Chrysostome Christophe A.A.M ${ }^{1}$ Et G. Apollinaire \\ Mensah. ${ }^{2}$ \\ ${ }^{1}$ Laboratoire de Recherche en Aviculture et en Zoo-économie (LARAZE), Faculté des Sciences Agronomiques (FSA) de \\ l'Université d'Abomey Calavi (UAC) 01 BP 509 Recette Principale, Cotonou 01,03 BP : 2070 Jéricho, Cotonou, \\ République du Bénin; Téléphone: +22995 585142 \\ ${ }^{2 *}$ Laboratoire des Recherches Zootechnique, Vétérinaire et Halieutique (LRZVH), Centre de Recherches Agricoles \\ d'Agonkanmey (CRA-Agonkanmey), Institut National des Recherches Agricoles du Bénin (INRAB), 01 BP 884 Recette \\ Principale, Cotonou 01, Tél. : (+229)97640947, République du Bénin \\ ${ }^{3}$ Direction Scientifique (DS/INRAB), 01 BP 884 Recette Principale, Cotonou 01, Tél. : (+229)97075465, Bénin. \\ Emails: cchrysostome@gmail.com, venuspascal69@gmail.com, fredericmb@gmail.com, begboris88@gmail.com.
} edenakpoa@gmail.com; mensahga@gmail.com,ga_mensab@yahoo.com,;ahoyonest@yahoo.com

* Auteur correspondant: Aimé Kocon EDENAKPO, edenakpoa@gmail.com

Mots clés : Complément alimentaire d'asticots, productivité, poule locale, Revenu, Bénin

Keywords: Food supplement of maggots, productivity, local hen, Income, Benin.

Publication date 31/08/2020, http://m.elewa.org/Journals/about-japs/

1 RESUME

Un complément alimentaire d'asticots frais a été servi pendant six mois aux poules locales élevées en divagation au niveau de 15 ménages d'aviculteurs traditionnels dans le village de Hessouhoué, commune d'Aplahoué au Sud-Ouest du Bénin. L'objectif est de tester en milieu réel l'effet des asticots sur la productivité des poulets en aviculture traditionnel. Pour ce faire, soixante (60) poules âgées de 25 semaines avec un poids moyen de $810 \mathrm{~g}$ et réparties en deux lots ont été élevées en divagation sous un traitement expérimental. Un lot de 30 poules constituant le premier groupe a reçu un complément d'asticots (TDACA) et le second lot sans complément d'asticots (TDSCA). À l'issue des analyses statistiques, les résultats n'ont montré aucune différence significative $(p>0,05)$ entre les deux traitements en ce qui concerne le nombre d'œufs pondus $(8,80$ œufs pour TDACA et 7,79 œufs pour TDSCA) et éclos (7,80 poussins pour TDACA et 6,54 poussins pour TDSCA) à la première ponte. Cependant, à la deuxième ponte, la moyenne d'œufs pondus $(10,31$ œufs) pour le TDACA est significativement plus élevées $(\mathrm{p}<0,05)$ que les 7,82 œufs obtenus pour le TDSCA. De plus, il n'existe aucune différence significative entre les poules concernant le taux d'éclosion pour les deux traitements (TDACA $=89,11 \%$ et TDSCA $=88,35 \%$ ). Les résultats montrent également que l'intervalle entre deux pontes pour le TDSCA (71,18 jours) est significativement plus élevé que celui du TDACA (66,28 jours). Il ressort de l'étude que l'utilisation des asticots frais en complément alimentaire, augmente le nombre d'œufs pondus par les poules en divagation, réduit l'intervalle entre deux pontes et améliore le revenu des aviculteurs. 
Influence of the dietary supplement of fresh maggots to wandering local hens on their productivity and on the poultry income of poultry farmers in South-West Benin.

\begin{abstract}
A dietary supplement of fresh maggots was served for six months to local chickens reared at 15 traditional farmer households in the village of Hessouhoué, a commune of Aplahoué in southwestern Benin. The objective was to test in a real environment the effect of maggots on the productivity of chickens in traditional poultry farming. To do this, sixty (60) 25 -weekold hens with an average weight of $810 \mathrm{~g}$ and divided into two groups were reared in stray under an experimental treatment. A batch of 30 hens constituting the first group received a supplement of maggots (TDACA) and the second batch without supplement of maggots (TDSCA). At the end of the statistical analyses, the results showed no significant difference $(p>0.05)$ between the two treatments with regard to the number of eggs laid $(8.80$ eggs for TDACA and 7.79 eggs for TDSCA) and hatched (7.80 chicks for TDACA and 6.54 chicks for TDSCA) at the first laying. In contrast to the second lay, the average of laid eggs (10.31 eggs) for TDACA was significantly higher $(p<0.05)$ than the 7.82 eggs obtained for TDSCA. In addition, there was no significant difference between hens in hatch rate for the two treatments $($ TDACA $=89.11 \%$ and TDSCA $=88.35 \%$ ). The results also showed that the spawning interval (71.18 days) was significantly higher than that for TDACA (66.28 days). The study found that the use of fresh maggots as a dietary supplement increases the number of eggs laid by free-range hens, reduces the interval between two layings and improves the income of poultry farmers.
\end{abstract}

\title{
2 INTRODUCTION
}

L'aviculture dispose de nombreuses potentialités parmi lesquelles la courte durée de son cycle de reproduction et de production, le retour rapide sur les investissements, la forte accessibilité à toutes les couches sociales etc., lui confèrent une place de choix dans les stratégies de développement et de lutte contre la pauvreté au Niger et dans la plupart des pays d'Afrique subsaharienne (MOOVE ARES, 2018). L'aviculture traditionnelle plus particulièrement, joue un rôle clé dans la sécurité alimentaire et contribue aux moyens d'existence des populations rurales sur les plans religieux, social et culturel (Alder, 2005; Ayissiwédé, 2013 ; Youssao et al., 2013). Selon ces mêmes auteurs, elle contribue aussi à l'amélioration des revenus des petits exploitants en milieu rural, notamment les femmes et sert de l'engrais organiques pour l'agriculture. Au Bénin, les poulets locaux domestiques représentent la principale ressource aviaire (Organisation des Nations Unies pour l'alimentation et l'agriculture [FAO], 2015).

L'élevage du poulet local est largement pratiqué en milieu rural et dans une moindre mesure en milieu urbain. C'est l'une des principales activités menées par les personnes disposant de peu de ressources (Vidogbena et al., 2010). Selon les statistiques de la Direction de l'Élevage du Bénin, le cheptel avicole est passé de 13 millions de poulets locaux en 2004 à 18 millions en 2014 (Direction de l'Élevage [DE], 2014) et constitue la deuxième source de viande (21\%), après les bovins (58\%) (Fanou, 2006). Par ailleurs, très prisée en Afrique au Sud du Sahara à cause des qualités physicochimiques et organoleptiques de sa viande, la volaille traditionnelle n'arrive pas encore à satisfaire les demandes des populations. Différentes contraintes (infrastructures, affections et maladies, alimentation, etc.) empêchent les petits aviculteurs d'obtenir de bonnes performances zootechniques et financières de leur élevage (Lwelamira, 2012 ; Ayssiwédé et al., 2013). L'alimentation apparaît comme l'une des contraintes majeures, avec notamment la non 
disponibilité, le prix élevé des ingrédients alimentaires protéiniques tels que la farine de poisson et le tourteau de soja (Ayssiwédé et al., 2013; Ouedraogo et al., 2015). Face à cette flambée du prix desprotéines conventionnelle, l'une des solutions envisagées aujourd'hui, est l'utilisation des sources alternatives de protéines dans l'alimentation de la volaille, par l'introduction des termites et asticots (Organisation des Nations Unies pour l'Alimentation et l'Agriculture [FAO], 2015). D'après les enquêtes effectuées par Pomalégni et al. (2016) au Bénin, les aviculteurs traditionnels ont une bonne connaissance des asticots, pensent que les larves de mouches améliorent la productivité des poulets et consentent majoritairement $(82 \%)$ à les valoriser dans l'alimentation des poulets locaux. Au Burkina Faso, Sankara (2017) rapporte que $47,8 \%$ des éleveurs connaissent les asticots. Toutefois, la production et l'utilisation effective des larves de mouches pour alimenter la volaille tarde à rentrer dans l'habitude des aviculteurs.

\section{MATERIEL ET METHODOLOGIE}

3.1 Milieu d'étude : L'étude a été réalisée à Hessouhoué dans la commune d'Aplahoué. La commune d'Aplahoué est située au Sud-Ouest du Bénin entre $6^{\circ} 56^{\prime}$ Latitude Nord et $1^{\circ} 41^{\prime}$ Longitude Est. Elle est caractérisée par un climat subéquatorial comprenant deux (02) saisons sèches (juillet à septembre et novembre
En effet, les études au Bénin comme au Burkina Faso (Pomalégni et al., 2016 ; Sankara, 2017) ont montré respectivement que seulement $5,73 \%$ et $12,8 \%$ des éleveurs utilisent les asticots. Mieux, peu de données expérimentales existent en milieu réel, pour confirmer ou infirmer la perception des aviculteurs sur l'importance des asticots dans l'alimentation de la volaille. Cette étude vise à démontrer aux aviculteurs traditionnels à travers des expérimentations dans leurs élevages, les avantages de la production et l'utilisation des larves de mouches pour une meilleure amélioration des performances productives des oiseaux. Pour y parvenir, une évaluation de l'effet du complément alimentaire à base d'asticots sur les performances de reproduction des poulets locaux a été réalisée à Hessouhoué, suivie d'une enquête socioéconomique sur le revenu avicole généré après utilisation des asticots en complément alimentaire chez les poulets auprès des aviculteurs ciblés.

à mars) et deux (02) saisons pluvieuses dont l'une courte (septembre à novembre) et l'autre plus longue (avril à juillet). Les précipitations annuelles varient de 900 à $1100 \mathrm{~mm} / \mathrm{an}$. Ces caractéristiques du climat subissent des modifications par moment à cause de certaines perturbations (Afrique Conseil, 2006). 

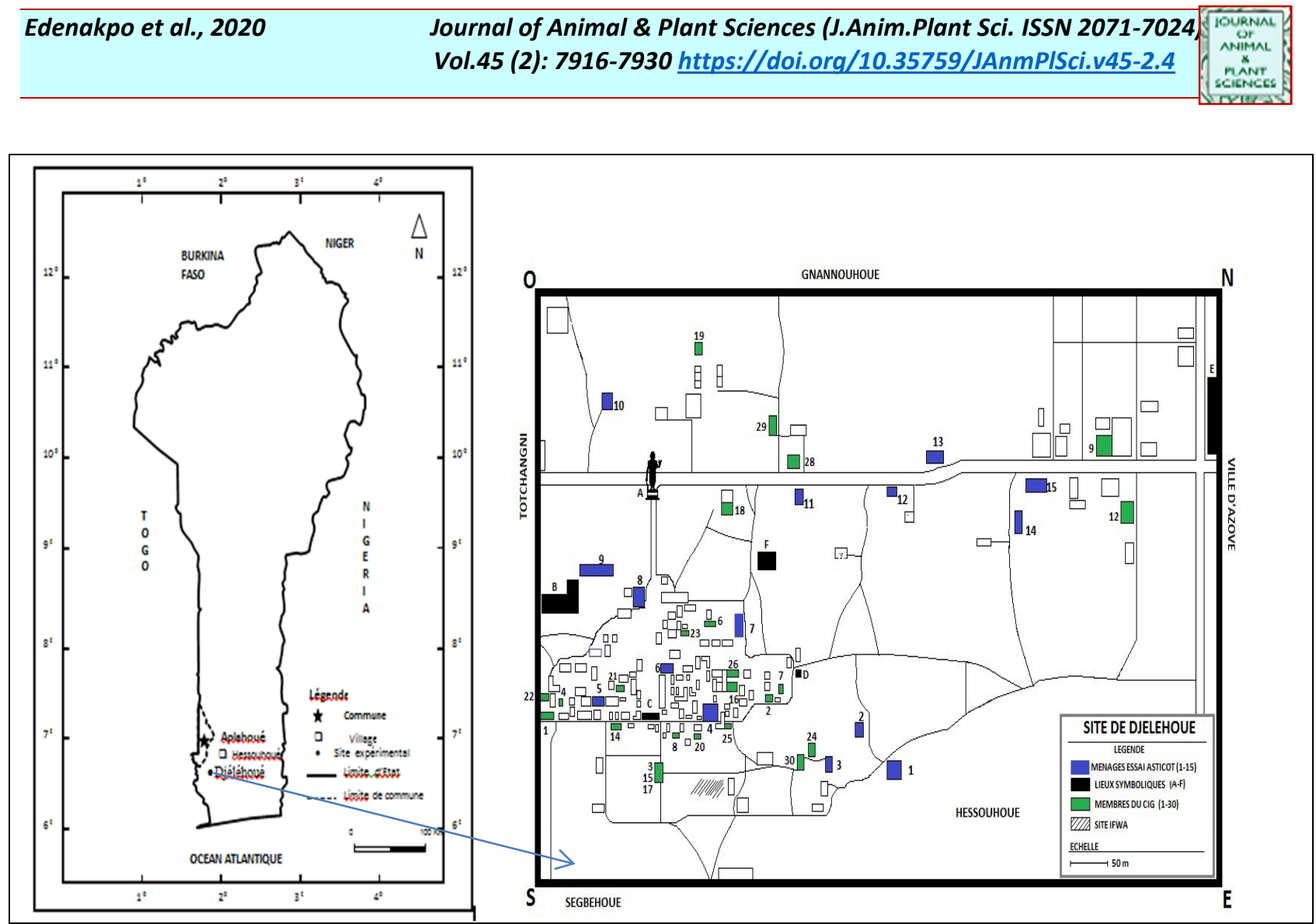

Figure 1 : Carte du site expérimental de Djéléhoué à Hessouhoué

Source : Données de terrain, 2019

L'étude a été réalisée en deux phases: une phase de suivi de productivité des poules en essai et une phase d'enquête sur le revenu avicole des aviculteurs. Pour ce qui concerne la phase de suivi de productivité, 60 poulettes de 25 semaines d'âge et pesant $810 \mathrm{~g}$ en moyenne ont été choisies dans 15 ménages d'aviculteurs traditionnels répondant aux critères de sélection des éleveurs devant abriter l'essai. Les critères de choix étaient : $\mathrm{i}$ - disposer d'au moins quatre (4) poulettes locales de même tranche d'âge et d'un coq local ; ii- disposer d'un abri de nuit et d'endroits sécurisés pouvant servir de nids aux poules; iii- n'avoir pas été formé sur la technique de production des asticots par le Projet "Insects as Feed in West Africa" (AFWA). Tous les poulets des ménages retenus ont été soumis à un plan prophylactique minimal (couverture vaccinale, déparasitage et antibiothérapie). Deux traitements ont été appliqués : i- traitement divagation sans complément d'asticots (TDSCA) c'est-à-dire le témoin et ii- traitement divagation avec complément d'asticots (TDACA). Les poules cherchaient la grande partie de leur aliment en divagation cependant, un complément de $30 \mathrm{~g}$ d'asticots frais (Dankwa et al., 2002) a été servi tous les matins avant huit (08) heures à deux (2) d'entre elles, les deux (2) autres étant des témoins (4 poules ayant servi à l'expérimentation par ménage). Les poules d'essai ont été servies sous un panier pour éviter la compétition avec les autres. Elles ont été libérées après la complémentation journalière d'asticots pour la divagation habituelle. Le test a été conduit pendant six (6) mois avec deux cycles de reproduction. Les larves de mouches utilisées ont été produites chaque jour dans l'asticoterie du village par le responsable de groupe d'implémentation du projet IFWA sur le site de Djéléhoué, avec des substrats locaux du village (Figure 2). La technique de production a été celle indiquée par (Pomalégni et al., 2016). Les asticots étaient récoltés tous les après-midi et distribués tôt les matins aux éleveurs pour leurs poules, afin de 
s'assurer que les oiseaux recevaient les mêmes quantités d'asticots tous les jours. Le mode de reproduction des poules était naturel. Le coût de production des asticots a été calculé à partir du prix du son de maïs qui était le substrat principalement utilisé et qui coûtait 12 FCFA/kg, livré par les femmes du village. Le temps de travail de la main d'œuvre et le coût des amortissements d'infrastructures et matériels de production ont été ajoutés aux coûts du substrat utilisé pour déterminer le coût de production d'asticots pendant l'essai. Ces données ont été encodées dans une base de données Excel. Le calcul économique après

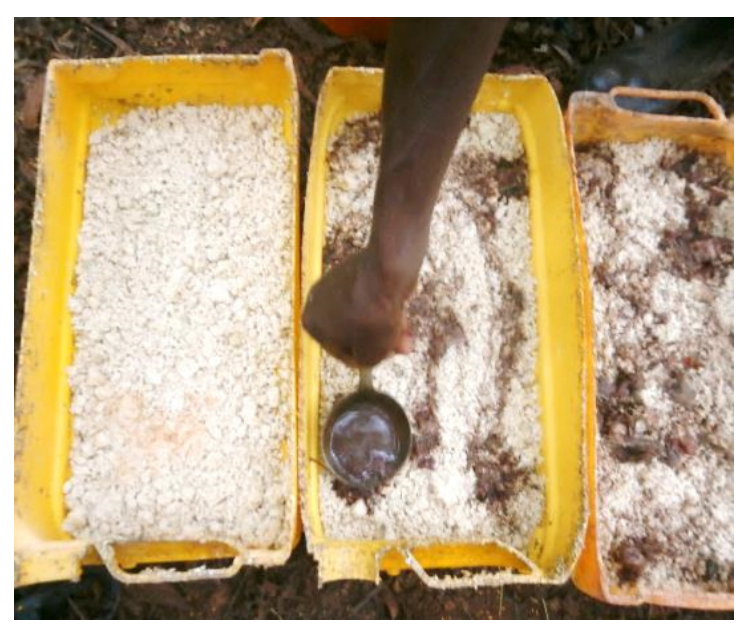

Photo 1: Ensemencement de substrat avec viscères de poissons (attractant mouches)

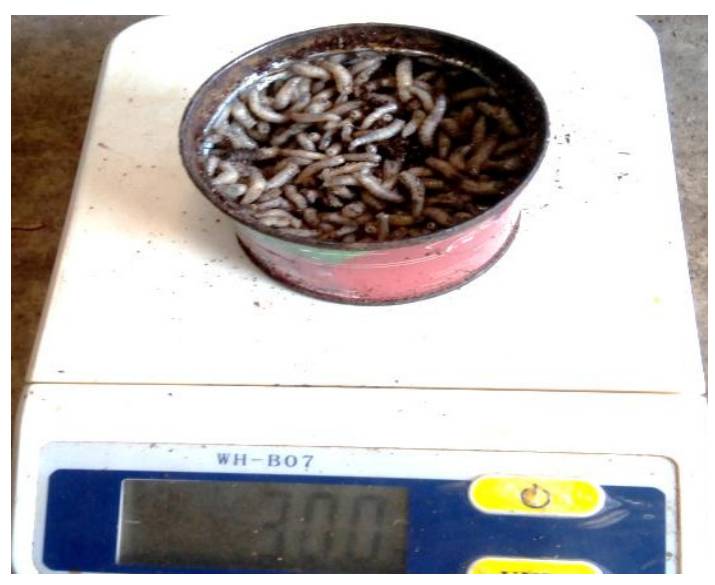

Photo 3 : Pesée de $30 \mathrm{~g}$ d'asticots frais l'introduction des asticots en complément aux poules a été fait en déduisant le coût de production d'asticotsservis, du revenu de vente des poulets dans la période d'essai chez l'éleveur. Une balance mécanique (pour peser les poulets), une balance électronique (pour peser les œufs et les asticots), des bagues en anneau numérotées (pour le marquage des poules), des paniers (pour protéger les poules expérimentales), des barques (bidons de 30 litres coupés longitudinalement en deux), des bassines, des tamis pour la production des asticots et un appareil photo ont été utilisés pour l'essai.

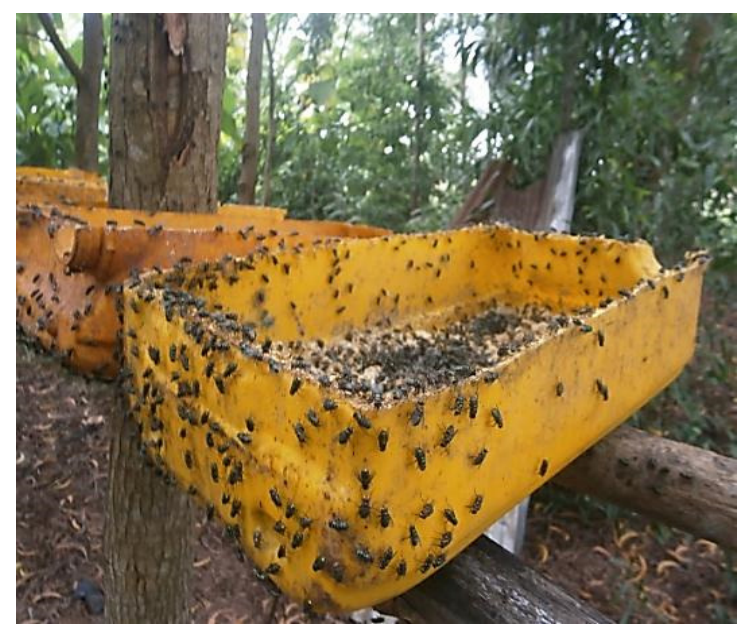

Photo 2: Exposition du substrat à l'oviposition naturelle

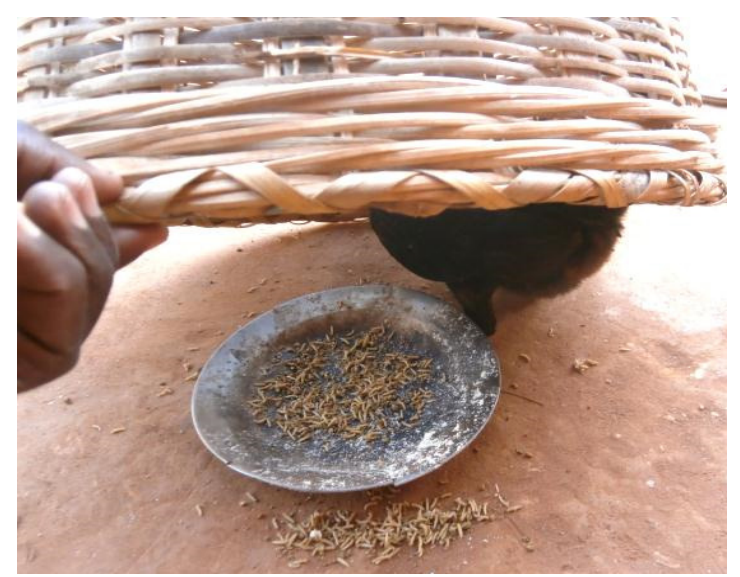

Photo 4 : Service d'asticots sous panier

Figure 2 : Étape de production et de service d'asticots aux poules 
Concernant l'enquête socioéconomique, elle a été conduite en trois (3) principales étapes à savoir: i- élaboration des fiches de collecte d'informations technico-économiques pour les enquêtés; ii- organisation d'une réunion avec les aviculteurs ayant abrité l'essai pour les informer sur le travail à faire par les enquêteurs; iii- l'administration du questionnaire à chaque éleveur dans son élevage. Les données collectées ont été encodées dans la base Excel pour la génération des tableaux et des graphiques qui ont été analysés et commentés.

3.3 Données collectées et paramètres calculés : Pour les données de reproduction, le nombre d'œufs pondus par poule par cycle, le nombre d'œufs fertiles, le nombre d'œufs éclos et le nombre de poussins vivants ont été enregistrés. Concernant les informations sur le revenu avicole, la productivité des poulets avant et après l'utilisation des compléments d'asticots a été recueillie chez les éleveurs et les calculs économiques ont été faits. Les paramètres calculés étaient: le taux d'éclosion, le poids moyen des œufs, le poids moyen des poussins et le nombre moyen d'œufs par poule par couvée.

3.4 Analyse statistique des données : Le test de Wilcoxon à 2 échantillons indépendants a été utilisé pour les paramètres tels que le poids moyen des œufs et le poids moyen des poussins. Pour le nombre d'œufs pondus, le nombre d'œufs éclos, le taux d'éclosion et le nombre de poussins vivants, un modèle linéaire généralisé de la famille poisson a été utilisé. Les données ont été analysées avec le logiciel $\mathrm{R}$ 3.5.1. Pour l'enquête sur le revenu avicole des éleveurs, le Test de normalité de Kolmogorov et Shapiro-Wilk a été utilisé pour vérifier la normalité des variables et le test $t$ de Student pour vérifier la significativité des différences entre le revenu avant et après l'introduction du complément d'asticots dans les élevages.

pondus par poule, le poids moyen des œufs, le nombre d'œufs couvés et le nombre d'œufs éclos au niveau des deux traitements. (Tableaux 1 et 2). $(\mathrm{p}>0,05)$ n'a été notée sur le nombre d'œufs

Tableau 1 : Ponte et couvaison des œufs par les poules suivant les traitements

\begin{tabular}{l|c|c|c}
\hline \multirow{2}{*}{ Variables } & \multicolumn{2}{|c|}{ Traitements } & \multirow{2}{*}{ Prob } \\
\cline { 2 - 3 } & TDACA & TDSCA & 0,18 \\
Nombre d'œufs pondus & 8,8 & 7,79 & 0,72 \\
Poids moyen des œufs & 30,08 & 30,44 & 0,171 \\
Nombre d'œufs couvés & 8,63 & 7,61 & 0,072 \\
Nombre d'œufs éclos & 7,8 & 6,54 & $0,014^{*}$ \\
\hline
\end{tabular}

* différence significative au senil de $5 \%$, TDSCA: traitement divagation sans complément d'asticots, TDACA: traitement divagation avec complément d'asticots.

Tableau 2 : Viabilité des poussins suivant les traitements

\begin{tabular}{l|c|c|c}
\hline \multirow{2}{*}{ Variables } & \multicolumn{2}{|c|}{ Traitements } & \multirow{2}{*}{ Prob } \\
\cline { 2 - 3 } & TDACA & TDSCA & $0,025^{*}$ \\
\hline Nombre de poussins vivants & 7,73 & 6,18 & 0,396 \\
\hline
\end{tabular}

* différence significative au seuil de $5 \%$, TDSCA: traitement divagation sans complément d'asticots, TDACA: traitement divagation avec complément d'asticots. 
4.1.2 Deuxième cycle de ponte: Au deuxième cycle de ponte, le nombre d'œufs pondus par poule, le nombre d'œufs couvés, le nombre d'œufs éclos et le nombre de poussins vivants sont significativement plus élevés $(p<$ $0,01)$ chez les poules expérimentales que chez les témoins (Tableaux 3 et 4). Concernant l'intervalle entre deux pontes, il était significativement plus bas $(66,28$ jours $)$ pour TDACA que pour TDSCA (71,18 jours). Le taux d'éclosion n'a présenté aucune variation selon les traitements $(p>0,05)$.

Tableau 3 : Ponte et couvaison des œufs par les poules suivant les traitements

\begin{tabular}{l|c|c|c}
\hline \multirow{2}{*}{ Variables } & \multicolumn{2}{|c|}{ Traitements } & \multirow{2}{*}{ Prob } \\
\cline { 2 - 3 } & TDACA & TDSCA & $0,002^{* *}$ \\
Nombre d'œufs pondus & 10,31 & 7,82 & $0,049 *$ \\
Poids moyen des œufs & 33,39 & 32,53 & $0,002^{* *}$ \\
Nombre d'œufs couvés & 10,31 & 7,82 & $0,002^{* *}$ \\
Nombre d'œufs éclos & 9,14 & 6,82 & 0,762 \\
Taux d'éclosion & 89,11 & 88,35 &
\end{tabular}

** différence significative au seuil de 1\%,TDSCA: traitement divagation sans complément d'asticots, TDACA: traitement divagation avec complément d'asticots.

Tableau 4 : viabilité des poussins suivant les traitements

\begin{tabular}{l|c|c|c}
\hline \multirow{2}{*}{ Variables } & \multicolumn{2}{|c|}{ Traitements } & \multirow{2}{*}{ Prob } \\
\cline { 2 - 4 } & TDACA & TDSCA & $0,001^{* *}$ \\
Nombre de poussins vivants & 9,00 & 6,61 & 0,225 \\
Poids moyens des poussins & 23,76 & 23,53 & 0,159 \\
Intervalle entre deux pontes & 66,28 & 71,18 & \\
\hline
\end{tabular}

** différence significative au seuil de 1\%, TDSCA : traitement divagation sans complément d'asticots, TDACA: traitement divagation avec complément d'asticots.

4.2 Coût de revient d'asticots utilisés par producteur pendant l'essai: Dans les tableaux 5, 6 et 7 ont été présentés le coût des amortissements du matériel utilisé, le coût du substrat et celui de la main d'œuvre pour la production du kilogramme d'asticots. Huit (08) kilogrammes en moyenne de son de maïs frais suffisaient pour produite un (1) kilogramme d'asticots frais dans cette expérimentation.

Tableau 5 : Coût de l'amortissement du matériel de production d'asticots frais sur le site IFWA de Hessouhoué

\begin{tabular}{l|c|c|c|c|c|c}
\hline Type & $\begin{array}{c}\text { Nombre } \\
\text { (NB) }\end{array}$ & $\begin{array}{c}\text { Prix } \\
\text { unitaire }\end{array}$ & Montant & $\begin{array}{c}\text { Durée } \\
\text { de vie }\end{array}$ & $\begin{array}{c}\text { Valeur } \\
\text { amortissement }\end{array}$ & $\begin{array}{c}\text { Coût par } \\
\text { production } \\
\text { (FCFA) }\end{array}$ \\
\cline { 3 - 4 } & & (FCFA) & (FCFA) & (ans) & & \\
Asticotérie & 1 & (PUF) & (MOT) & (DV) & & 2,74 \\
Barques & 20 & 300 & 6000 & 2 & 3000 & 8,22 \\
Bassines & 2 & 800 & 1600 & 2 & 800 & 2,19 \\
Tamis & 4 & 1500 & 6000 & 1 & 6000 & 16,44 \\
Peson & 1 & 5000 & 5000 & 2 & 2500 & 6,85 \\
\hline Total 1 & & & & & & 36 \\
\hline
\end{tabular}


Tableau 6 : Coût des consommables de production d'asticots frais sur le site IFWA de Hessouhoué

\begin{tabular}{l|c|c|c|ll}
\hline \multirow{2}{*}{ Type } & Quantité & Prix unitaire & Montant & Coût total/kg & d'asticots \\
\cline { 2 - 5 } & $(\mathrm{kg})$ & $($ FCFA) (PUF) & (FCFA) (MOT) & (FCFA) & \\
Son de maïs & 8 & 12 & 96 & 96 & \\
Déchets de poisson & 1 & 33 & 33 & 33 & \\
$\begin{array}{l}\text { Divers déplacements } \\
\text { Honoraire de }\end{array}$ & 1 & 50 & 50 & 50 & \\
production & 8 & 1200 & 225 & 225 & \\
\hline Total 2 & & & & & $\mathbf{4 0 4}$ \\
\hline
\end{tabular}

Tableau 7 : Coût total de consommation d'asticots par éleveur en 6 mois

\begin{tabular}{l|c}
\hline Elément de coût & Montant (FCFA) \\
\hline Total 1 & 36 \\
Total 2 & 404 \\
Coût total du kilogramme d'asticots frais & 440 \\
Coût mensuel de production d'asticots frais & 13213 \\
Coût de production d'asticots frais en 6 mois & 79279 \\
Coût de consommation d'asticots frais par éleveurs en 6 mois & 5285 \\
\hline
\end{tabular}

4.3 Analyse des données d'enquête sur l'utilisation des asticots en complément alimentaire aux poulets chez les aviculteurs 4.3.1 Répartition des enquêtés selon le sexe : La figure 3 illustre la répartition par sexe des éleveurs enquêtés. De l'analyse de la figure, il ressort que plus de trois quart des enquêtés sont des femmes contrairement aux hommes qui occupent une faible proportion de l'échantillon considéré.

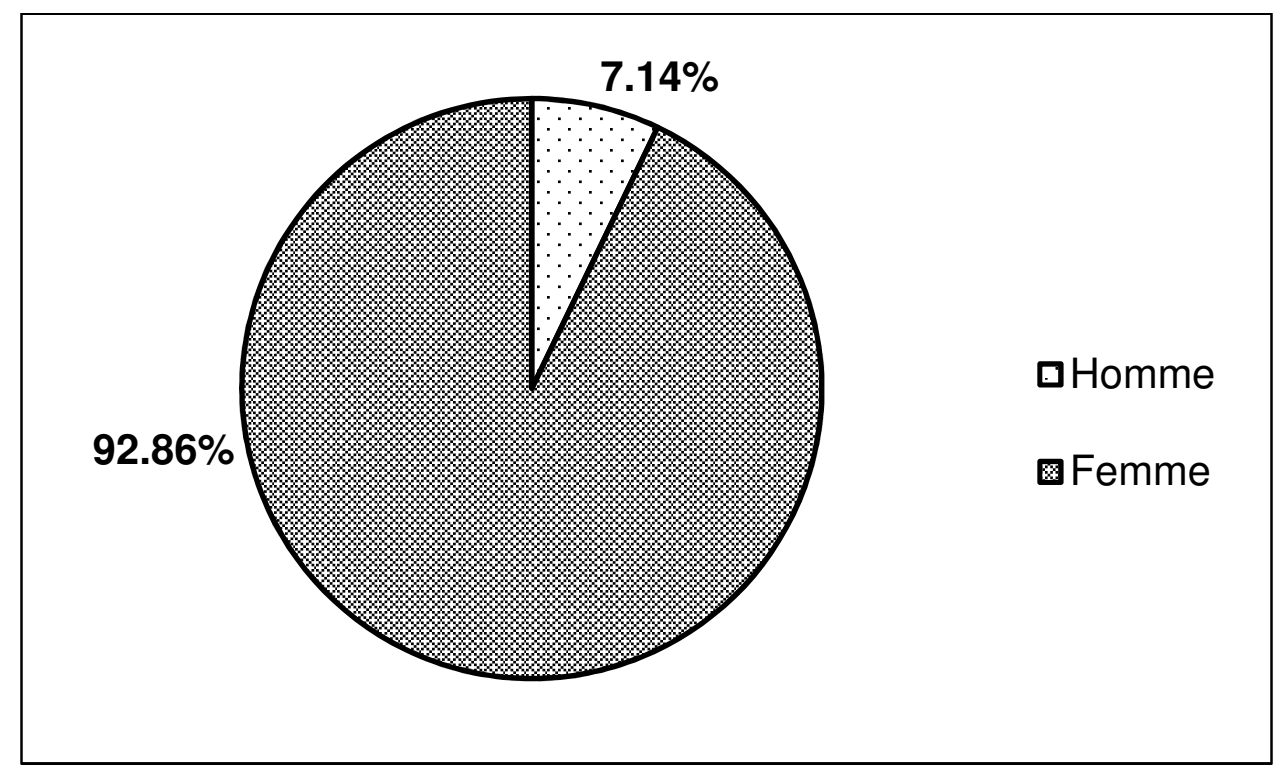

Figure 3 : Répartition des enquêtés selon le sexe Source : Données de terrain, 2019 
4.3.2 Répartition des enquêtés selon qu'ils soient instruits ou non : La figure 4 renseigne que sur l'ensemble des éleveurs enquêtés,
$71,43 \%$ d'entre eux ne sont pas instruits, contrairement à 28,57\% qui sont allé à l'école.

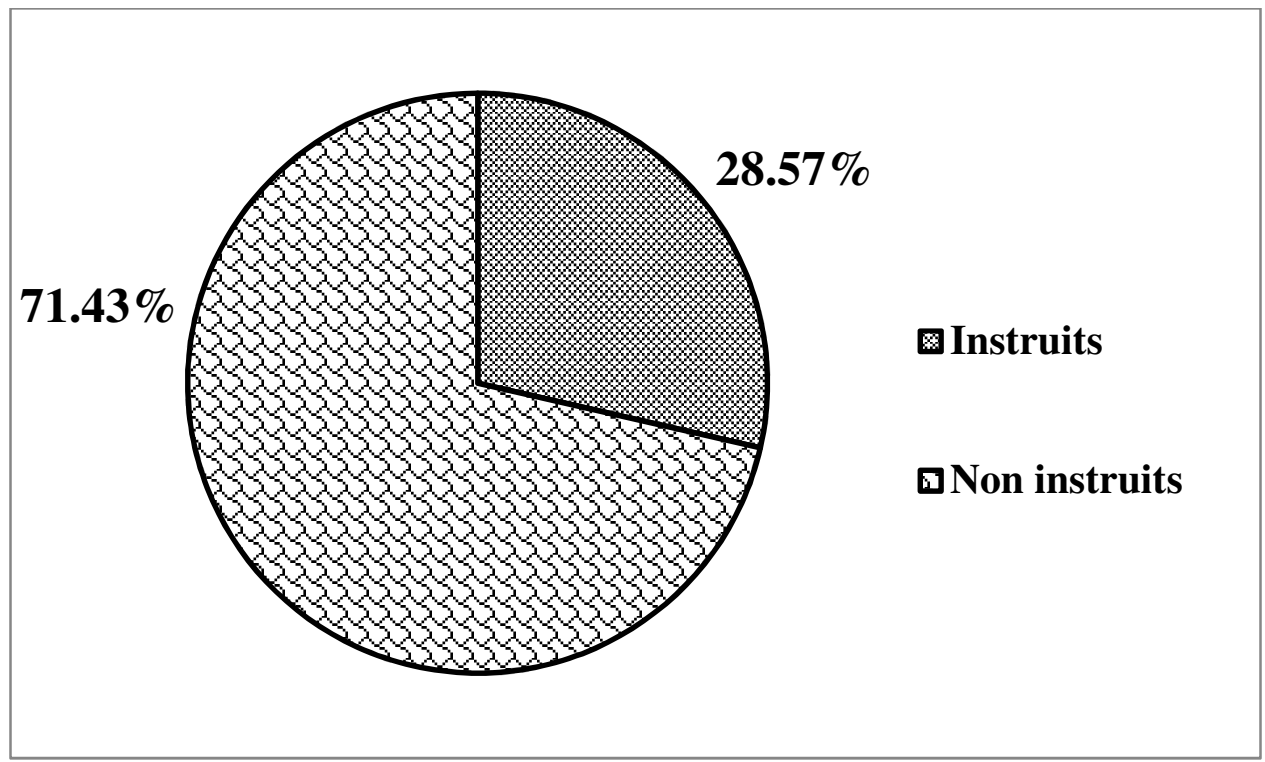

Figure 4 : Situation d'instruction des enquêtés

Source : Données de terrain, 2019

Interaction entre le sexe et l'activité principale des aviculteurs traditionnels est illustrée dans la figure 5

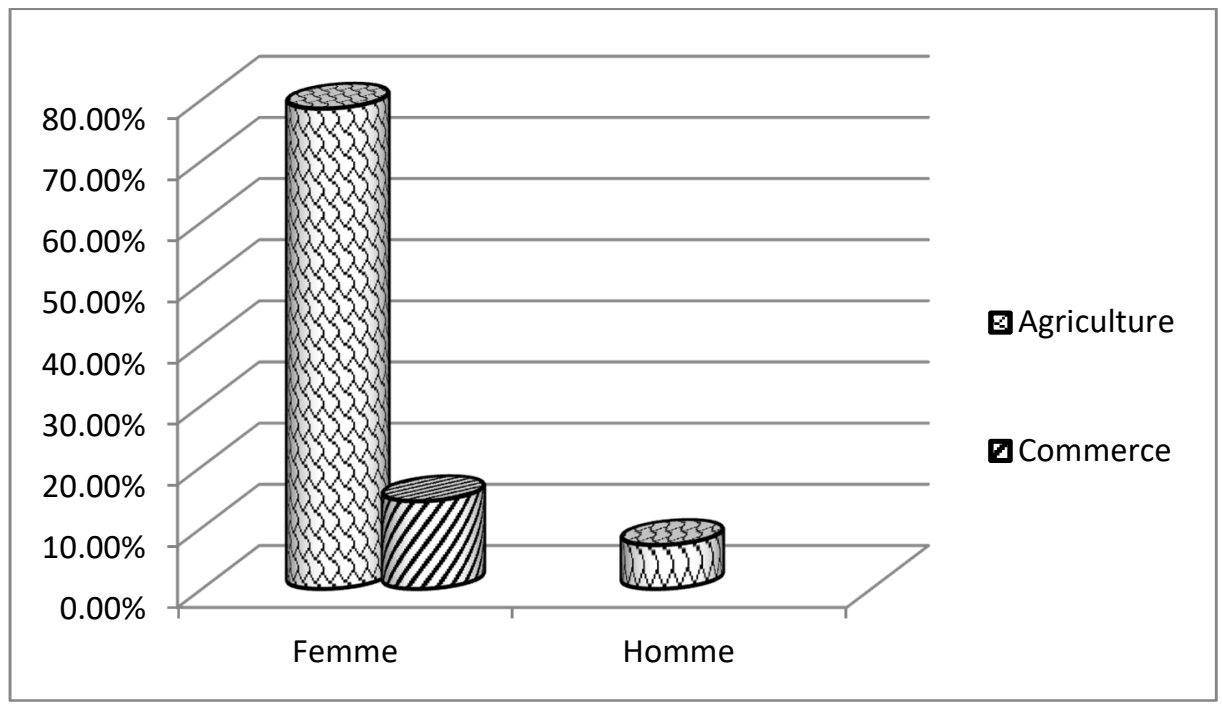

Figure 5 : Distribution du sexe selon l'activité principale des enquêtés

Source : Données de terrain, 2019

Il ressort de cette figure que les femmes sont majoritaires dans les deux principales activités identifiées. Le secteur de l'agriculture domine chez les aviculteurs. Les trois quart des acteurs sont des femmes contrairement aux hommes qui ne constituent qu'un cinquième des enquêtés. Le tableau 8 illustre l'analyse descriptive de la productivité économique des poulets avant et après l'utilisation des asticots par les éleveurs 
Tableau 8 : Analyse descriptive de la productivité économique des poulets

\begin{tabular}{l|l|l|l|l}
\hline Intitulé & Moyenne & Ecart-type & Minimum & Maximum \\
\hline Revenu net/après l'utilisation de l'asticot & 27850,71 & 19660,617 & 0 & 58115 \\
Revenu net/ avant l'utilisation de l'asticot & 19762,50 & 11529,022 & 0 & 35650 \\
\hline
\end{tabular}

Source : Données de terrain, 2019

Les résultats du tableau (8) montrent qu'en moyenne le revenu net après l'utilisation des asticots est supérieur à celui avant l'utilisation des asticots. Cependant, ces résultats n'apportent pas assez d'information sur les significativités de la différence entre les deux moyennes. Les résultats du test de corrélation (Tableau 9) montrent qu'il existe une relation significative entre les deux variables $(p<0,05)$.

Tableau 9 : Corrélations pour échantillons appariés

\begin{tabular}{|c|c|c|c|c|}
\hline & & $\mathrm{N}$ & Corrélation & Sig. \\
\hline Paire 1 & Revenu/av \& Revenu net/ap & 14 & 0,869 & 0,000 \\
\hline
\end{tabular}

Revenu/av $=$ revenu avant utilisation d'asticots Revenu net/ap $=$ revenu net après utilisation d'asticots

Analyse de la normalité des variables : Les résultats (Tableau 10) montrent que la probabilité associée est supérieure à 5\%. Ainsi, on ne peut pas rejeter l'hypothèse nulle de normalité. Les variables de revenu avant utilisation des asticots et le revenu net après utilisation des asticots suivent une loi normale. Les résultats du test de Shapiro-Wilk montrent également que les variables suivent une loi normale.

Tableau 10 : Tests de normalité

\begin{tabular}{l|c|c|c|c|c|c}
\hline & \multicolumn{3}{|c|}{ Kolmogorov-Smirnov $^{\text {a }}$} & \multicolumn{3}{c}{ Shapiro-Wilk } \\
\cline { 2 - 7 } & Statistique & ddl & Signification & Statistique & ddl & Signification \\
\hline Revenu net/ap & 0,144 & 14 & $0,200^{*}$ & 0,950 & 14 & 0,561 \\
\hline Revenu/av & 0,120 & 14 & $0,200^{*}$ & 0,947 & 14 & 0,510 \\
\hline
\end{tabular}

*. Il s'agit d'une borne inférieure de la signification réelle. a. Correction de signification de Lilliefors

\section{Analyse de la différence significative à partir du t de Student}

Tableau 11 : Test des échantillons appariés

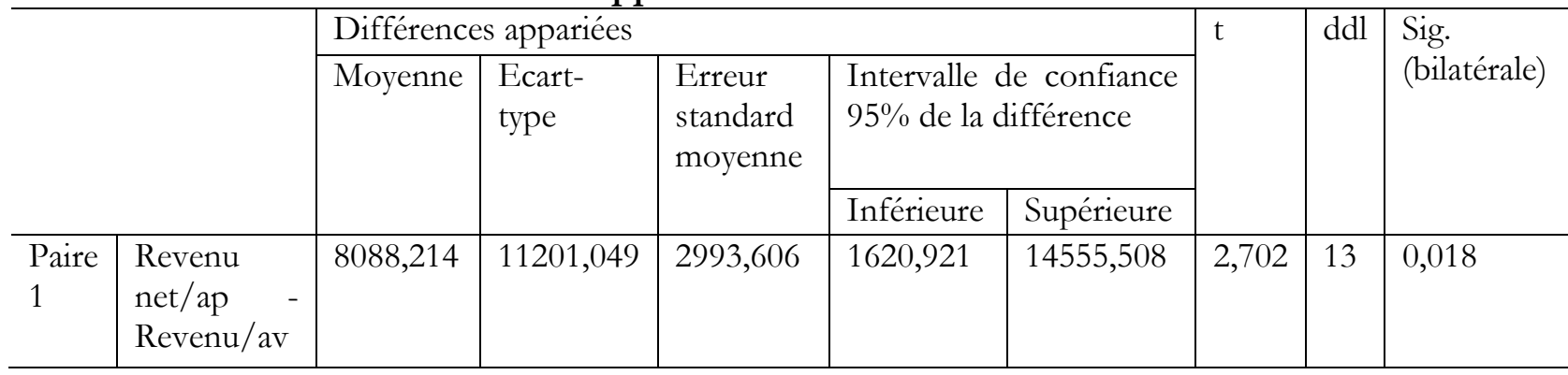

La différence de la moyenne entre les revenus avant et après l'utilisation des asticots a été de 8088,214 avec un écart-type de 1120,049. La probabilité associée à la différence des moyennes a été de 0,018 montrant ainsi une différence significative entre le revenu avant et après l'utilisation des asticots. 


\section{DISCUSSION}

5.1 Productivité des poules locales nourries avec un complément d'asticots

5.1.1 Nombre moyen d'eufs pondus et poids moyen des aufs : Le nombre moyen d'œufs pondus par les poules dans les deux traitements de cette expérimentation se retrouve dans les intervalles de ponte signalés par plusieurs auteurs (Msoffe et al., 2004 ; Akouango et al., 2010 ; Ayssiwédé et al., 2013). Il reste cependant légèrement inférieur aux moyennes signalées respectivement pour les phénotypes fauves, caillouté et froment dans la revue de la FAO (2015) au Bénin. Toutefois, la différence significative notée à la deuxième ponte entre les poules ayant reçu un complément d'asticots frais et les témoins se justifie par l'apport nutritif des larves de mouches. En effet, les poules des deux traitements étant laissées en divagation, l'apport des asticots aux oiseaux d'un traitement constitue pour eux un supplément alimentaire quantitatif et qualitatif. Sur le plan qualitatif, l'asticot frais de par sa composition chimique notifiée par Đordevic et al. (2008 a apporté des éléments nutritionnels (protéines, calcium, phosphore) pour stimuler la ponte chez les poules complémentées. Les mêmes observations ont été faites au Kenya sur l'amélioration significative des performances de croissance et de productivité des oiseaux supplémentés par l'apport de $8 \mathrm{~g}$ d'un aliment concentré protéique à la ration de base des poulets traditionnels en divagation (Kingori et al. 2007). Nos résultats concordent également avec ceux d'Agunbiadé et al. (2007) et confirme ainsi le rôle des larves de mouches dans l'amélioration du taux de ponte chez les poules traditionnelles. Mieux, Maurer et al. (2016), en remplaçant totalement le tourteau de soja par la farine de larve de mouche du genre Hermetia dans l'alimentation des poules pondeuses Lohmann Brown de 64 semaines d'âge d'essai ont obtenu une production de 5,4\% au-dessus de celle du groupe témoin. L'amélioration du ratio phosphocalcique par les asticots et qui est un élément favorisant la ponte chez les poules est également prouvée par Marono et al. (2017).
Pomalégni (2017) a rapporté le rôle stimulateur de ponte de ce rapport, lorsqu'il a obtenu la ponte précoce chez les poules nourries avec les asticots dans son expérimentation. Ainsi la production des asticots permet non seulement le recyclage rapide des déchets organiques dans le village d'étude, mais aussi l'obtention de la biomasse de larves de mouches qui apportent efficacement des nutriments pour améliorer la productivité des poulets.

\subsubsection{Taux d'éclosion et viabilité des} poussins : a pratique de l'élevage avicole dans le village d'étude, couplée à l'aptitude des poules indigènes à être de bonnes couveuses (Ayssiwédé et al., 2013) justifient les taux d'éclosion obtenus. Ces taux sont plus élevés aux taux moyens signalés par de nombreux auteurs (Sonaiya et Swan, 2004 ; Msoffe et al., 2004 ; Akouango et al., 2010) en couvaison naturelle. En effet, les éleveurs pour des raisons de sécurité, enferment les poulets la nuit dans leur cuisine ou dans leur chambre. Les poulets étant habitués à ces endroits qu'elles trouvent sécurisés y pondent leurs œufs. Elles obtiennent ainsi de bonnes conditions d'incubation, à l'abri des intempéries (pluie, humidité, soleil etc.), des prédateurs et des parasites qui affectent le taux d'éclosion. La viabilité élevée des poussins du lot des poules nourries à base des asticots par rapport à celle des poussins du lot témoin se justifie par la consommation des larves de mouches qui a impacté positivement l'organogenèse des poussins. Cette observation concorde avec celle de Edénakpo et al. (2020) qui ont trouvé une bonne viabilité des poussins chez les poules nourries avec la ration à base de $11 \%$ de farine d'asticots. Les résultats rapportés par Bouvarel et al. (2010) confirment nos résultats quant au poids de l'œuf conditionné par la concentration énergétique de l'aliment, les teneurs en protéines et acides aminés (méthionine notamment) et en acides gras (acides linoléique et oléique). Ainsi, les œufs étant enrichis en nutriments par les asticots, ont permis d'avoir des poussins vigoureux, plus résistants aux maladies et donc plus viables que les poussins du lot témoin. Nos observations 
corroborent également les résultats de Đordevic et al. (2008) et de Hwangbo et al. (2009) qui ont prouvé que la substitution de la farine de poisson par les asticots frais ou séchés a un effet positif sur les performances de production des oiseaux et sur leur état de santé. Cet avantage alimentaire des asticots sur les poules s'est fait remarquer également au niveau de l'intervalle entre deux pontes. En effet, après l'éclosion, les poules complémentées ont commencé plus tôt une nouvelle ponte que les témoins. Ce qui veut dire qu'elles ont récupéré assez vite leur poids perdu lors de la couvaison. Cette observation concorde avec celle de Sarkar et Bell (2006). Autrement, si l'utilisation des larves de mouches est continue sur les poulets, le nombre de cycle de reproduction va augmenter comme l'ont estimé Edénakpo et al. (2020), améliorant ainsi la productivité numérique des oiseaux. Les asticots, plus accessibles aux aviculteurs traditionnels que la farine de poisson, apportent aux poulets des éléments nutritifs indispensables pour la reproduction et la croissance des oiseaux.

\subsection{Productivités numérique et} économique des poules en divagation complémentées avec des asticots frais selon les enquêtés: En ce qui concerne la productivité des poules nourries aux asticots, le constat des producteurs sur l'amélioration de la ponte vient confirmer les résultats de l'essai en

\section{CONCLUSION}

Cette étude a montré que la production des larves de mouches pour alimenter la volaille est possible en aviculture familiale, et ceci suivant la disponibilité des substrats et de l'adaptabilité de la méthode de production aux conditions du milieu des éleveurs. L'étude a montré aussi la possibilité d'utiliser les asticots produits comme principale source de protéines animales dans les rations en remplacement des ressources protéiniques animales ou végétales usuelles plus coûteuses et difficiles d'accès aux éleveurs comme la farine de poisson et le tourteau de soja. L'utilisation de ces larves de mouches en complément alimentaire aux oiseaux dans le présent travail a donné de meilleurs résultats sur milieu réel et concorde avec les résultats d'enquête réalisée chez les aviculteurs du Burkina Faso par Sankara (2017). Sur le plan socio-professionnel, la tendance observée avec la dominance des femmes dans l'agriculture et le commerce se justifie par leur représentativité largement supérieure à celle des hommes dans l'échantillon enquêté. En effet, le sous-secteur d'activité choisie pour l'étude (l'aviculture familiale) est majoritairement pratiqué par les femmes dans le village. Cette remarque de la dominance des femmes dans l'aviculture concorde avec celle de nombreux auteurs (Alder, 2005; Ayissiwédé, 2013; Sankara, 2017). Sur le plan économique, l'augmentation sensible du revenu avicole des éleveurs enquêtés provient de l'amélioration de la productivité numérique des poules soumises à l'utilisation des asticots. Ce revenu était utilisé pour payer la tontine, le petit déjeuné aux enfants et pour régler la popote en cas de difficulté financière au sein du ménage. Ces déclarations concordent avec celles des auteurs qui confèrent des rôles socioculturel et économique indéniables à l'aviculture traditionnelle (Arkar, 2006; Fotsa, 2008; Ayissiwédé, 2013). Toutefois, les aviculteurs trouvent que leur revenu aurait été plus élevé avec les asticots s'ils arrivent à maitriser la conduite des poussins avant et après leur sevrage par les poules-mères.

leur reproduction, avec plus d'œufs pondus et de poussins sevrés, favorisant ainsi l'amélioration de la productivité du cheptel en aviculture familiale. Ainsi, l'augmentation sensible du cheptel des aviculteurs traditionnels impliqués dans l'étude a impacté significativement leur revenu. L'existence des substrats de production en milieu rural, la possibilité d'utiliser la forme fraiche des asticots et la simplicité de la technique de production des larves sont autant d'opportunités susceptibles de faciliter l'adoption de cette pratique par les aviculteurs. Il serait cependant judicieux de reprendre l'étude avec une ration complète à bases des ingrédients localement 
disponibles afin de mettre au point une formule alimentaire à base des asticots au coût accessible aux petits aviculteurs. Ceci permettrait une utilisation optimale des larves

\section{REMERCIEMENTS}

Cette étude a été réalisée avec l'appui financier du projet "Insects as Feed in West Africa" (IFWA). Les auteurs remercient Dr David DJIMENOU et MSc BONOUGBO Hamdy pour leurs contributions à la rédaction de l'article, M. Cossi FAGNINOU, Responsable de mouches qui est une alternative de choix face aux coûts sans cesse croissant des aliments conventionnels utilisés en aviculture.

du Groupe d'Implémentation du projet IFWA à Hessouhoué pour son soutien indéfectible dans la collecte des données, M. Simon Oladé EDENAKPO et tous les producteurs ayant participé à la réalisation de cette étude.

\section{REFERENCES BIBLIOGRAPHIQUES}

Afrique Conseil: 2006. Monographie de la commune d'Aplahoué. 50p.

Agunbiade A.J, Adeyemi O.A, Ashiru O.M, Awojobi H.A., Taiwo A.A, Oke D.B and Adekunmisi A.A : Replacement of fish meal with maggot meal in Cassavebased layers' diets. The Journal of Poultry Science, 44: 278-282.

Akouango F., Bandtaba P. et Ngokaka C: 2010. Croissance pondérale et productivité de la poule locale Gallus domesticus en élevage fermier au Congo. Anim. Genet. Resour, 46: 61-65.

Alders R. : 2005. L'aviculture : source de profit et de plaisir. Brochure de la FAO sur la diversification, Rome. $3: 1-39$.

Arkar K. S and Bell J.G : 2006. Potentiel du poulet indigène et son rôle dans la lutte contre la pauvreté et dans la sécurité alimentaire pour les ménages ruraux. Bull. RID AF, 16: 16-28.

Ayssiwédé S.B., Dieng A., Houinato M.R.B., Chrysostome C.A.AM., Issay I., Hornick J-L. and Missohou A: 2013. Elevage des poulets traditionnels ou indigènes au Sénégal et en Afrique Subsaharienne : état des lieux et contraintes. Ann. Méd. Vét. 157 : 103119.

Bouvarel I., Y. Nys, M. Panheleux et Lescoat P : 2010. Comment l'alimentation des poules influence la qualité des œufs ? In : Numéro Spécial, Qualité de l'œuf. Nys Y. (Ed). Inra Prod. Anim., 23 : 167-182.
Dankwa, D., Nelson, F.S., Oddoye, E.O.K., J.L Duncan: 2002. Housefly larvae as a feed supplement for rural poultry. Ghana J. Agric. Sci. 35, 185-187.

Direction de l'Élevage: 2014. Annuaires statistiques. 31p.

Đordevic M., Radenkovic-Damnjanoviæ B. Vuciniæ M. Baltic M. Teodorovic R. Jankovic L. Vukasinovi M. and Rajkovic $M:$ 2008. Effects of substitution of fish meal with fresh and dehydrated larvae of the house fly (Musca domestica L.) on productive performance and health of broilers. Acta Vet. 58: 357-368.

Edenakpo K. A., Ahoyo Adjovi N. R., Pomalegni S. C. B., Anato C. H., Amagnide A., Aboh A. B., Chrysostome C. A. A. M. et Mensah G. A: 2020. Influence des rations alimentaires a base d'asticots sur la reproduction des poulets locaux au Bénin. Agronomie Africaine 32: 1 - 14 (sous presse).

Fanou U: 2006. Premières évaluations de la structure et de l'importance avicole, commercial et familial de l'Afrique de l'Ouest: Cas du Bénin; Rapport Organisation des Nation-Unies pour l'Alimentation et l'Agriculture, FAO. $32 \mathrm{p}$.

FAO : 2014. Secteur Avicole Sénégal. Revues nationales de l'élevage de la division de la production et de la santé animales de la FAO. Rome 7 : 78p. 
FAO : 2015. Secteur Avicole Bénin. Revues nationales de l'élevage de la division de la production et de la santé animales de la FAO, Rome. 10: 1-74

Fotsa J-C: 2008. Caractérisation des populations de poules locales (Gallus gallus) au Cameroun. Thèse de doctorat, Agroparistech, Paris. 301p.

Hwangbo J., Hong E.C., Jang A., Kang H.K., Oh J.S., Kim B.W. and Park B.S : 2009. Utilization of house fly-maggots, a feed supplement in the production of broiler chickens. J Environ. Biol. 30: 609-614.

IFWA: 2016. Étude de référence du projet "Insects as feed in West Africa" au Bénin. 71p. Dépôt légal N 9103 du 14 décembre 2016, 4ème trimestre, Bibliothèque Nationale (BN) du Bénin ISBN : 978-99919-2-661-2.

Kingori A. M., Tuitoek J.K., Muiruri H. K., Wachira A. M. and Birech E. K: 2007. Protein intake of growing indigenous chicken on free-range and their response to supplementation. International Journal of Poultry Science 6: 617-621.

Lwelamira, J., 2012: Phenotypic and genetic parameters for body weights and antibody response against Newcastle disease virus (NDV) vaccine for Kuchi chicken ecotype of Tanzania under extensive management. Trop. Anim. Health Prod. DOI 10.1007/s11250-0120099-z.

Marono S., Loponte R., Lombardi P., Vassalotti G., Pero M. E., Russo F., Gasco L., Parisi G., Piccolo G. and Nizza S : 2017. Productive performance and blood profiles of laying hens fed Hermetia illucens larvae meal as total replacement of soybean meal from 24 to 45 weeks of age. Poultry Science, 96: 1783-1790.

Maurer V., Holinger M., Amsler Z., Früh B., Wohlfahrt J., Stamer A. and Leiber F: 2016. Replacement of soybean cake by Hermetia illucens meal in layer hens.
Journal of Insects as Food and Feed, 2: 83-90.

Msoffe P.L.M., Mtambo M.M.A., Minga U.M., Olsen J.E., Juul-Madsen H.R., Gwakisa P.S., Mutayoba S.K. and Katule A.M : 2004. Productivity and reproductive performance of the free-range local domestic fowl ecotypes in Tanzania. Livest. Res. Rural Dev., 16. URL: http://www.lrrd.org//rrd16/9/msof160 67.htm 2016.

MOOVE ARES, 2018: Consommer local et promouvoir le poulet bicyclette ! Amélioration de la filière avicole dans la région de Niamey 2018 - 2022. Projets académiques et scientifiques de coopération au développement menés par l'ARES et ses partenaires. https://moove.ares-ac.be/niger/conso mmer-local-et-promouvoir-le-pouletbicyclette

Pomalegni S. C. B., Gbemavo D. S. J. C., Kpade C. P., Babatounde S., Chrysostome C. A. A. M., Koudande O. D. Kenis M. Glele K. R. L. et Mensah G. A : 2016. Perceptions et facteurs déterminant l'utilisation des asticots dans l'alimentation des poulets locaux (Gallus) au Bénin. Journal of Applied Biosciences 98 : 9330 - 9343.

Pomalégni S.C.B : $2017 . \quad$ Perception, performances zootechniques et qualité nutritionnelle de la viande de poulets locaux (Gallus gallus) nourris avec des rations alimentaires à base de larves de mouche (Musca domestica, Linnaeus 1758) au Bénin. Thèse de doctorat. Ecole des Sciences Agronomiques. Faculté des Sciences Agronomiques. Université d'Abomey-Calavi. 266p. Dépôt légal N9813 du 29/11/2017, Bibliothèque Nationale du Bénin, $4^{\text {ème }}$ trimestre. ISBN 978-99919-72-99-2.

Sankara F: 2017. Co-construction de techniques de production, d'extraction et de séchage de larves de mouche domestique à l'Ouest du Burkina Faso. Mémoire de fin de cycle en vue de 
l'obtention du diplôme d'Ingénieur du Développement Rural, option vulgarisation agricole, $69 \mathrm{p}$.

Sonaiya E.B. and Swan S.E: 2004. Production en aviculture familiale: un manuel technique. Organisation des Nations Unies pour l'Alimentation et l'Agriculture, FAO/Productions et santé animales, Rome, Italie. 134p.

Vidogbena F., Adegbidi A., Garnett S.T., Koudande D., Agbo V., Zander K : 2010. Peace, health or fortune? Preferences for chicken traits in rural Benin. Ecological Economics, 69: 1848-1857.

Youssao A.K.I, Tougan U.P., Ahounou S.G., Houessionon B.F.J. and Koutinhouin B: 2013. Typology of local poultry breeding of Gallus gallus species in family poultry in Benin. International Journal of Agronomy and Agricultural Research $4: 1-13$ 